\title{
Early warning signals also precede non-catastrophic transitions
}

\author{
Sonia Kéfi, Vasilis Dakos, Marten Scheffer, Egbert H. Van Nes and Max Rietkerk \\ S. Kéfi (sonia.kefi@univ-montp2.fr), Inst. des Sciences de l'Evolution, CNRS UMR 5554, Univ. de Montpellier II, Place Eugène Bataillon, \\ CC 065, FR-34095 Montpellier, Cedex 05, France. - V. Dakos, M. Scheffer and E. H. Van Nes, Dept of Aquatic Ecology and Water Quality \\ Management, Wageningen Univ., PO Box 47, NL-6700AA Wageningen, the Netherlands. VD also at: Estación Biologica de Doñana, \\ C/Américo Vespucio S/N, ES-41092 Sevilla, Spain. - M. Rietkerk, Dept of Environmental Sciences, Utrecht Univ., PO Box 80115 , \\ NL-3508 TC Utrecht, the Netherlands.
}

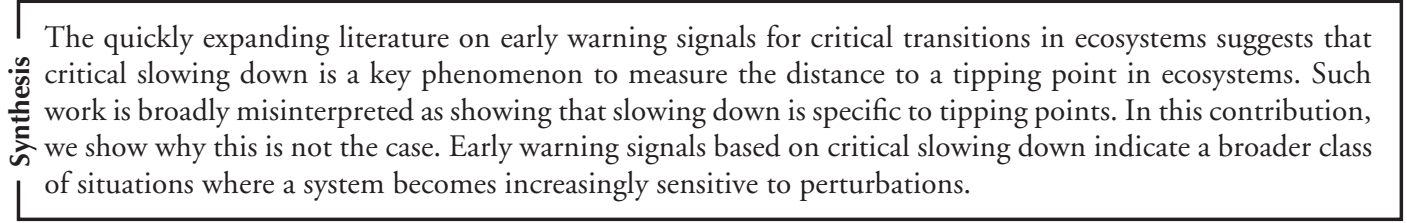

Ecosystem responses to external changes can surprise us by their abruptness and irreversibility. Models have helped identifying indicators of impending catastrophic shifts, referred to as 'generic early warning signals'. These indicators are linked to a phenomenon known as 'critical slowing down' which describes the fact that the recovery rate of a system after a perturbation decreases when the system approaches a bifurcation - such as the classical fold bifurcation associated to catastrophic shifts. However, contrary to what has sometimes been suggested in the literature, a decrease in recovery rate cannot be considered as specific to approaching catastrophic shifts. Here, we analyze the behavior of early warning signals based on critical slowing down in systems approaching a range of catastrophic and non-catastrophic situations. Our results show that slowing down generally happens in situations where a system is becoming increasingly sensitive to external perturbations, independently of whether the impeding change is catastrophic or not. These results highlight that indicators specific to catastrophic shifts are still lacking. More importantly, they also imply that in systems where we have no reason to expect catastrophic transitions, slowing down may still be used in a more general sense as a warning signal for a potential decrease in stability.

Human footprint on ecosystems is growing, and ecosystems cope with these changes in different ways. Climatic changes or human activities may push some ecosystems to shift abruptly to a different state in an often irreversible manner. Such catastrophic shifts can even happen when external changes are gradual, in which cases the shifts are even harder to foresee. Because these radical changes in ecosystem states can coincide with loss of species, habitats and ecosystem services and might fundamentally affect the livelihood of people who depend on these resources, understanding the ecological mechanisms behind catastrophic shifts has attracted a lot of attention in ecology (Holling 1973, May 1977, Rietkerk et al. 1996, Scheffer et al. 2001, Folke et al. 2004). More generally, a broad range of complex systems, such as financial markets, human societies, and the climate, seems to exhibit abrupt (e.g. financial crashes, revolutions and hurricane) rather than gradual behavior in face of gradual external changes (Sornette 2002, 2003, Diamond 2004, Peters and Pielke 2004). Lately, the possibility of radical changes stimulated research targeting at identifying indicators - or 'catastrophe flags' in the words of Gilmore (1981) - that would allow us to assess the imminence of these abrupt, unexpected and irreversible shifts (Sornette 2002, Scheffer et al. 2009).

The search for indicators has been quite fruitful and has shown that the way ecosystem characteristics, such as biomass or population abundance, vary in space and time may give us hints about an approaching shift. In particular, ecosystems become slower when approaching a shift, meaning that close to a shift the return time back to equilibrium after a disturbance becomes longer. This phenomenon is called 'critical slowing down', and the family of indicators derived from it has been referred to as 'generic early warning signals' (reviewed by Scheffer et al. 2009). These indicators may be helpful not only for ecosystems but also for the wider range of complex systems previously mentioned that are likely to exhibit shift behaviors.

The search for indicators has mostly focused on model systems that exhibit a catastrophic shift, prior to which specific behaviors of the system were identified as potential early warning signals. The objective was to ultimately use these signals to detect catastrophic shifts in real ecosystems. 
However, ecosystems do not always respond in an abrupt, unexpected and irreversible (i.e. catastrophic) way. Instead, ecosystem changes may be smooth, gradual, and reversible (i.e. non-catastrophic). Nevertheless, it is not clear whether the early warning indicators observed before a catastrophic shift are also observed before other types of transitions (Van Nes and Scheffer 2007, Chisholm and Filotas 2009, Kuehn 2011). If they do, we are not dealing with generic early warning signals 'specific' to catastrophic shifts. Such specific indicators would be of high practical relevance because of the possibly considerable economic and ecological losses associated with catastrophic shifts in ecosystems.

In this paper, our aim is to test whether early warning signals also precede non-catastrophic transitions. We do this by comparing the behavior of the two main early warning signals (namely variance and autocorrelation) in ecosystem models before catastrophic and non-catastrophic shifts.

\section{Methods}

As a starting point, we used a simple model of overharvesting (Noy-Meir 1975, May 1977). This model is a classical example describing the occurrence of catastrophic shifts in ecosystems. The model describes the growth of a resource (e.g. vegetation) and its consumption (e.g. by grazers). As grazing pressure increases, the resource growth cannot sustain the losses due to grazing and the resource decreases. An interesting characteristic of this model is that the way in which the resource decreases in response to increasing grazing pressure (i.e. the type of transition) depends on the carrying capacity of the resource, $K$ (Table 1). Here, we tuned $K$ to obtain three different types of transitions: the classical catastrophic transition (fold bifurcation, for $K=10$ ), a sharp but continuous transition (cusp bifurcation, for $K=5.2$ ), and a gradual smooth response (no bifurcation, for $K=2$ ).

Additionally, we examined two other types of transitions related to non-catastrophic bifurcation points that are common in ecosystem models. For example, in the classical Lotka-Volterra competition model (Lotka 1925), species coexist up to a point at which interspecific competition drives one species extinct through a transcritical bifurcation. We used the overharvesting model described above (Noy-Meir 1975, May 1977) with a linear grazing functional response to study a transcritical bifurcation where increasing grazing leads to the extinction of the resource.

The last non-catastrophic transition we studied was the Hopf bifurcation. This transition describes the onset

Table 1. Model equation and parameter name, interpretation and values.

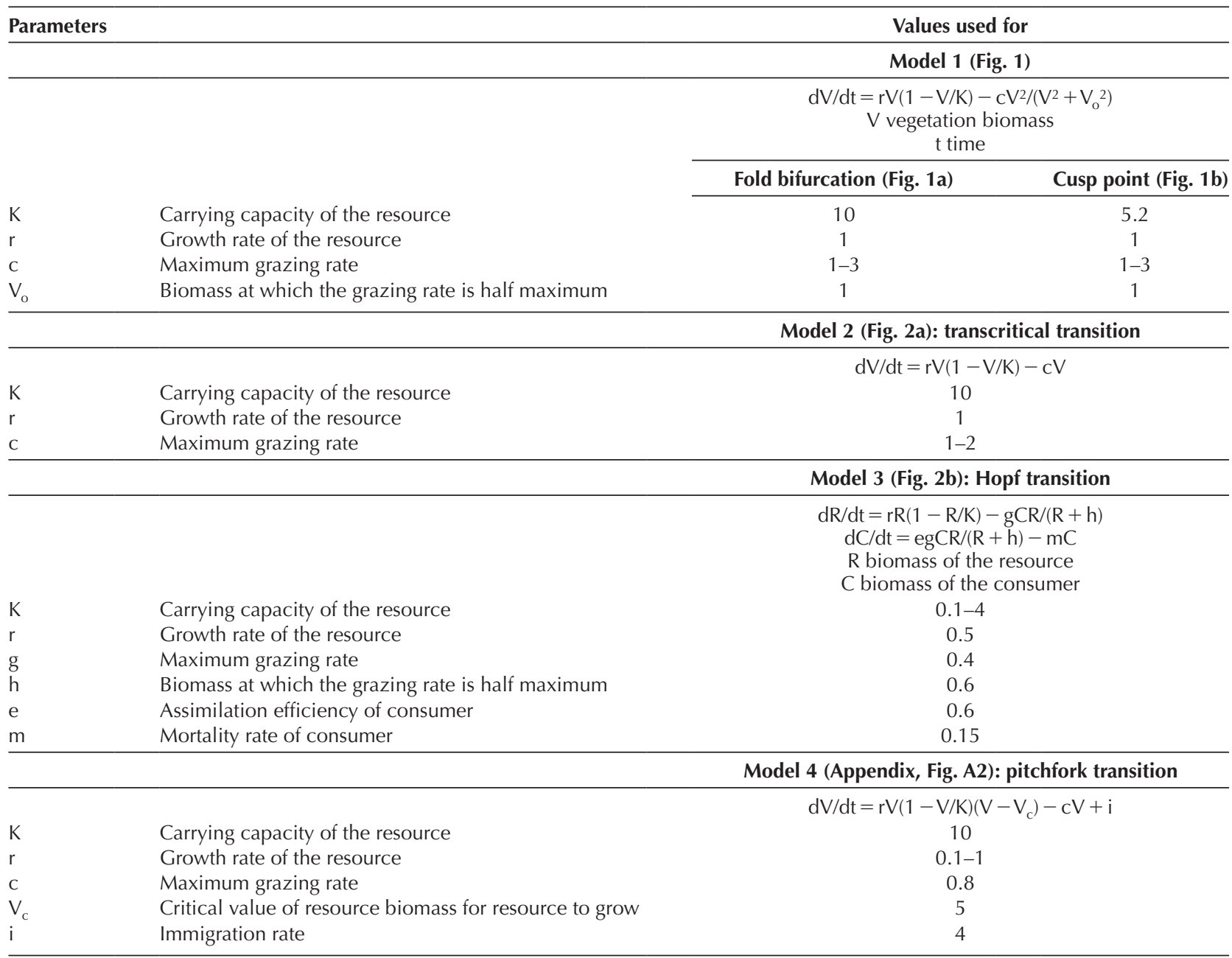


of oscillations in an otherwise stable population. It is a common transition described in consumer-resource models, where an increase in resource productivity drives the system into acyclic behavior, such as the well-studied predatorprey cycles of algae-zooplankton (Scheffer et al. 1997) and of hare-lynx (Stenseth et al. 1997). To study this bifurcation, we used the classical Rosenzweig-MacArthur model with a type II functional response (Rosenzweig 1971).

All models and the parameter values used for each type of transition are summarized in Table 1 . We chose a set of models that are classic in ecology for exhibiting a certain type of transition. However, the mathematical properties of a system going through these types of critical transitions (Kuehn 2011) is largely independent of the specific model formulation. Therefore our results should be independent of model specifications.
We estimated eigenvalues along all transition types using GRIND in MATLAB (available at < www.dow.wau.nl/aew/ grind/ $>$ ). We also followed changes in temporal autocorrelation and variance. To this end, for each level of the control parameters, we estimated the autocorrelation at-lag- 1 and the variance as the standard deviation of the state variable using 200 points of the produced time-series and using the same sequence of additive noise for each level of the control parameter. We only dealt with the scenario of a constant regime of noise prior to the transition. False alarms or deviations in the behavior of leading indicators prior to catastrophic transitions have been reported elsewhere in the case of non-constant regimes of noise or multiplicative noise (Brock and Carpenter 2010, Dakos et al. 2012). We expect that the same conclusions will also hold for the non-catastrophic cases as well. Autocorrelation at-lag-1 was estimated as the autoregressive coefficient from fitting a
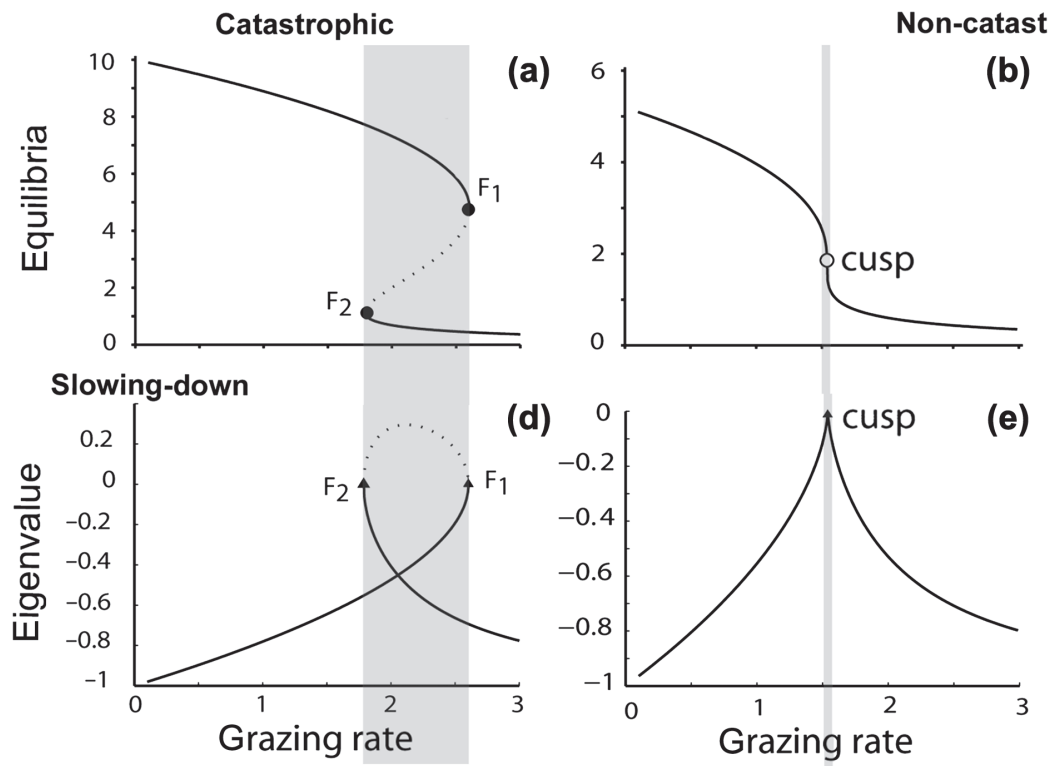

(b)
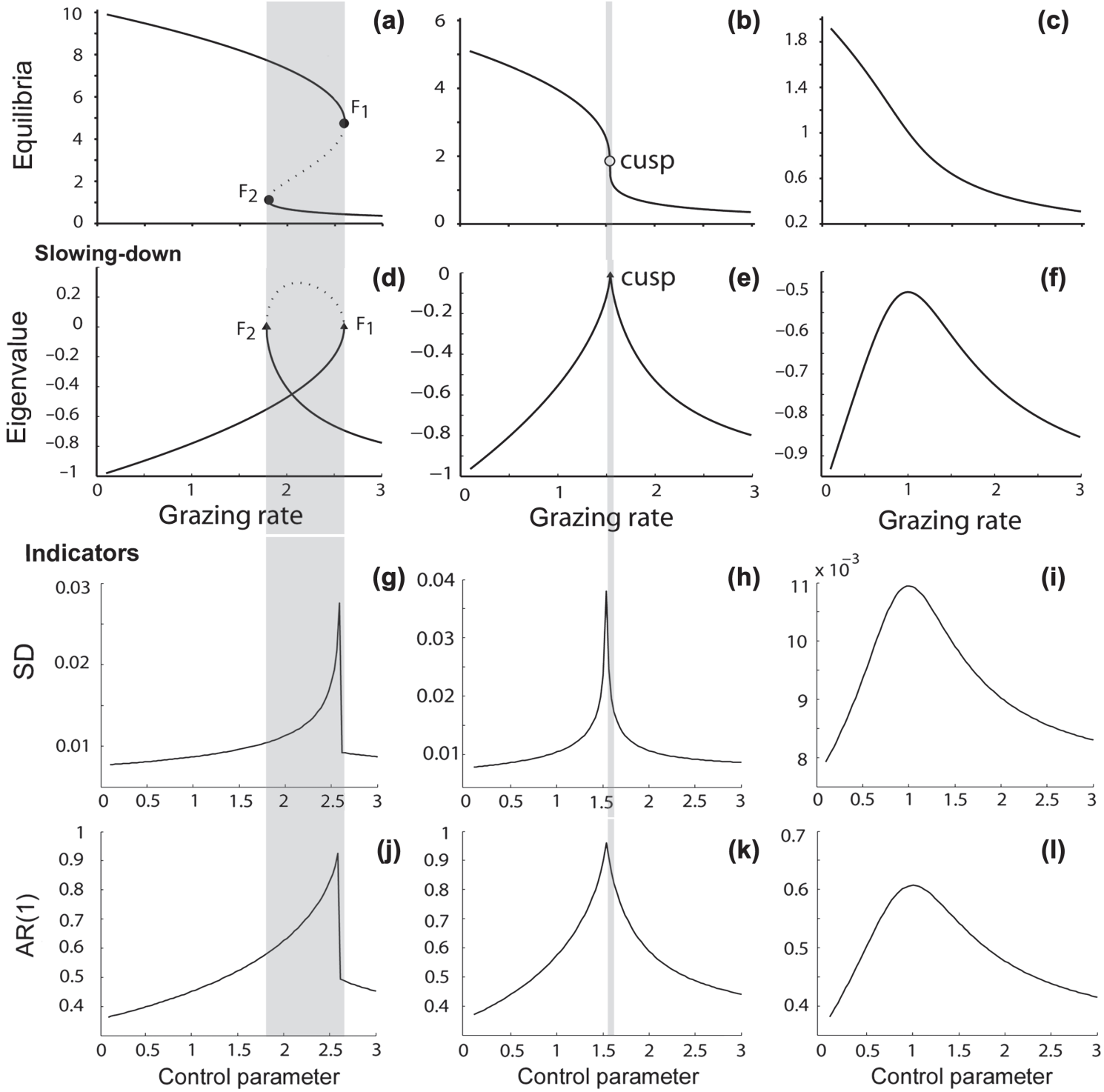

Figure 1. Behavior of the indicators along catastrophic vs non-catastrophic transitions in the overharvesting model (Noy-Meir 1975, May 1977). Left: fold bifurcation (abrupt, discontinuous), middle: cusp bifurcation (abrupt, continuous), right: no bifurcation (gradual, continuous). First row: model equilibria depending on the control parameter ( $c$; see Table 1 for parameter definition). Second row: dominant eigenvalue of the system. Third row: standard deviation. Fourth row: autocorrelation at lag one. All models and the parameter values used for the simulations are summarized in Table 1. 
linear autoregressive model of first order $\left(\mathrm{AR}(1), x_{t+1}-\mu=\right.$ $a_{1}\left(x_{t}-\mu\right)+\varepsilon$, where $\mathrm{a}_{1}$ is the autoregressive coefficient of order $1, \mu$ the mean of the timeseries and $\varepsilon$ an error i.i.d term) to the data. We solved the stochastic equations using Euler-Murayama integration with Ito calculus.

\section{Slowing down before a catastrophic transition}

What happens near a catastrophic shift? Several indicators that can signify proximity to a catastrophic shift have been recently suggested by simple models. A description of how these indicators change in the vicinity of catastrophic shifts can be found in Scheffer et al. (2009). Here, we will briefly illustrate these changes in the case of Noy-Meir's classical overharvesting model (Noy-Meir 1975). When grazing pressure increases, the vegetation biomass gradually decreases until the grazing pressure reaches the point $F_{1}$, referred to as the bifurcation point or tipping point (Fig. 1a). At this point the system collapses to a low biomass state, and a catastrophic change in the system's state occurs. Once the system has collapsed, recovery is possible only if the grazing pressure is decreased below another bifurcation point $F_{2}$, a phenomenon referred to as hysteresis, which is due to the coexistence of two alternative system's states between $F_{1}$ and $F_{2}$. In the rest of the paper, a 'catastrophic shift' refers to a fold bifurcation as illustrated in Fig. 1a. Note that the use of the word 'catastrophic' comes from the field of 'catastrophe theory' initiated by the mathematician René Thom, and refers to the abruptness of a system's response to small changes in external conditions and not to the possible negative consequences of the shift. A catastrophic shift can indeed have negative (e.g. shift from high to low biomass $F_{1}$ ) or positive (e.g. shift from low to high biomass at $F_{2}$ ) consequences. Hysteresis is what makes catastrophic transitions potentially difficult to reverse, or even irreversible, which is why indicators aiming at avoiding the occurrence of catastrophic shifts would be of great practical relevance.

When a catastrophic shift (or a fold bifurcation) is approached, it has been shown that a system becomes slow in returning to equilibrium after a perturbation. Mathematically, this phenomenon of critical slowing down (Box 1) is related to the fact that the real part of the dominant eigenvalue of the system goes to zero at a bifurcation point (Fig. 1d) (Wissel 1984, Strogatz 1994, Schefferet al. 2009). Critical slowing down translates into two other phenomena: 1) an increase in the system's variability (i.e. the standard deviation of temporal fluctuations in the state variable) and 2) an increase in the autocorrelation of the time series (Fig. 1g, j, Box 1). Interestingly, the spatial equivalents to those temporal indicators (i.e. spatial variance and spatial auto-correlation) increase as well when the system approaches a catastrophic shift (Oborny et al. 2005, Guttal and Jayaprakash 2009, Dakos et al. 2010).

\section{Slowing down before a non-catastrophic transition}

\section{Non-catastrophic transitions with no bifurcation}

While the fold bifurcation (Fig. 1a) represents a catastrophic ecosystem response due to the existence of alternative ecosystem states, there are cases where ecosystems respond sharply but not catastrophically to changing external conditions, in which case we talk about 'non-catastrophic' transitions. Figure $1 \mathrm{~b}$ illustrates a sharp but continuous decrease in biomass along a grazing gradient. Along this type of transitions, the eigenvalue goes almost to zero as the system approaches the cusp point, just like in the case of a catastrophic bifurcation (Fig. 1e). This implies that slowing-down is also to be expected (Strogatz 1994, Kuehn 2011, Lim and Epueranu 2011, Thompson and Sieber 2011). Indeed, we find that both variance and autocorrelation rise along the transition (Fig. 1h, k). This type of ecosystem response represents a common case of abrupt, nonlinear but continuous change in ecosystem states. However, it is a limit case to the fold bifurcation, and it is therefore not surprising that the indicators respond similarly as before a fold bifurcation (i.e. as in Fig. 1g, j).

What is more interesting is to see what happens along a transition that shows a smooth, gradual decrease in vegetation biomass (Fig. 1c). In this case, the eigenvalue does not reach zero. Nonetheless it reaches a maximum value at some point along the transition (Fig. 1f). This leads variance and autocorrelation to show similar trends as along the other transitions (Fig. 1i, l). Their increase is, however, not as steep as in the cases where the system exhibits a bifurcation point in our numerical examples (Fig. 1g, h, j, k).

\section{Non-catastrophic transitions with bifurcation}

What happens along other types of transitions that are associated with bifurcation points? Besides the fold, other types of bifurcations are commonly observed in ecological models. In the case of a transcritical bifurcation (Fig. 2a), a stable and an unstable equilibrium collide and exchange stability at the bifurcation point (Fig. A1 in Supplementary material Appendix A1). In the case of a Hopf bifurcation (Fig. 2b), a stable equilibrium is replaced by a stable limit cycle at the bifurcation.

At both transcritical and Hopf bifurcations, the eigenvalues reach zero (Fig. $2 \mathrm{c}-\mathrm{d}$ ). Indeed, we also find that the indirect indicators of critical slowing down, variance and autocorrelation of the state variables, both peak at the bifurcation points (Fig. 2e-h) in the same way as it happens along the transitions studied in Fig. 1. The same phenomenon occurs at other bifurcation types (Thompson and Stewart 2002, Thompson and Sieber 2011), such as the very uncommon pitchfork bifurcation (Strogatz 1994; Fig. A2 in Supplementary material Appendix A1). Thus, no difference in the behavior of the generic early warning signals prior to the fold, transcritical, and Hopf bifurcations is to be expected. However, it is noteworthy that the behavior of the state variables along these transitions may be more informative and help discriminating between these three types of transitions. For example, before the fold bifurcation, there are usually unnoticed changes in the state of the system, while before the transcritical bifurcation at least one state variable clearly goes to zero. In the case of the Hopf bifurcation damped oscillations in the state of the system are observed before the transition.

\section{Discussion}

In the case of ecosystems, but also more generally of complex systems (Sornette 2002, 2003, Peters and Pielke 


\section{Box 1. What happens near a catastrophic shift?}

\section{Basic theory}

A now classic analogy to understand how an ecosystem may respond to an increasing pressure is the one of a ball rolling in a landscape (Noy-Meir 1975, May 1977, Gilmore 1981). The landscape represents a collection of possible states for the ecosystem. The ball rolls down the slope and settles at the bottom of the valleys, which represent the stable states. More technically, the landscape is the 'potential' of the system in physics and can be calculated for Noy-Meir's model.

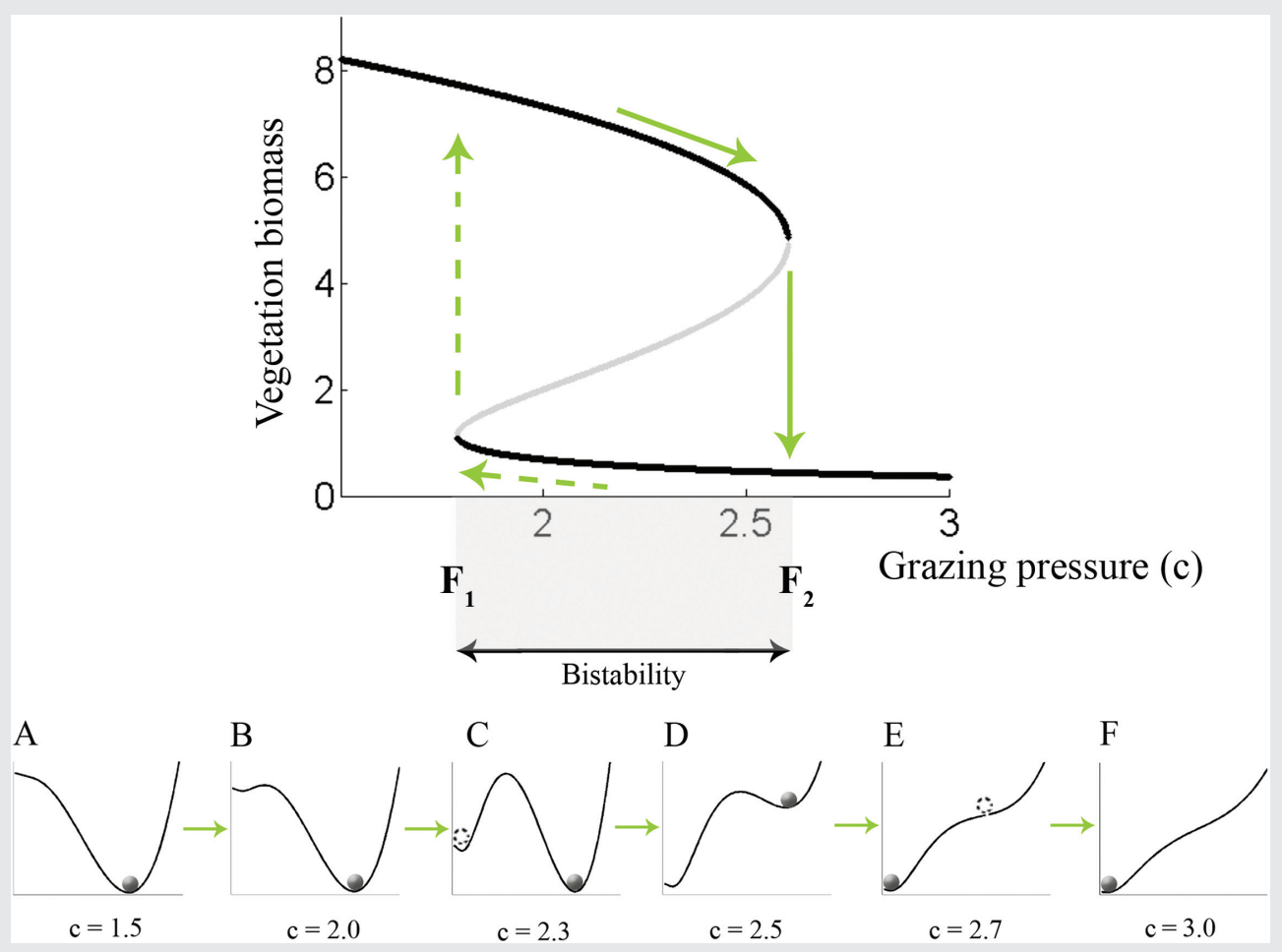

Figure I. Fold bifurcation (equation and parameter values are in Table 1). The curve represents the equilibrium vegetation biomass as a function of the grazing rate, $c$. Black lines correspond to stable states and grey lines to unstable states. Critical thresholds of the environmental conditions, $F_{1}$ and $F_{2}$, bound the parameter space where two stable states coexist, i.e. the bistability area. The two stable states are separated by an unstable one. Panels A-F display the potential function (or landscape) of the system for different values of $c$.

When grazing pressure increases, modifications of the shape of the landscape occur (Fig. IA-F). For small values of $c$, the landscape has only one valley. The system has a unique stable state of high biomass to which it inevitably tends toward from all initial conditions and following any disturbance (Fig. IA). When $c$ increases, a second valley appears (at $F_{1}$; Fig. IB-E). The system has now two equilibria, one corresponding to a high biomass and the other to a low biomass state. The valley into which the ball will settle depends on its initial location in the landscape. At the bifurcation point $F_{2}$, the first valley disappears and the landscape is again reduced to one valley. This is how the system collapses from a high to a low biomass state, i.e. where the catastrophic shift occurs.

Following more specifically the changes in the shape of the valley allows understanding more in detail the dynamics of the system as a shift is approached. The shape of the valley flattens (Fig. I, compare panels C, D and E). Because of this flattening of the landscape, the characteristic return time to equilibrium after a perturbation increases when the system gets closer to the threshold $F_{2}$, this means that a disturbed ecosystem needs more time to recover when it is nearer to a threshold (Gilmore 1981, Wissel 1984, Van Nes and Scheffer 2007). This phenomenon is referred to as 'critical slowing down' in the physics literature.

Two other phenomena follow from the flattening of the landscape. First, the system variability increases near a threshold (Gilmore 1981). It is indeed intuitive that a ball slightly pushed will move more in a flatter landscape. This is reflected by the increase in the standard deviation of temporal fluctuations in the state variable just before a transition occurs (Oborny et al. 2005, Carpenter and Brock 2006) (Fig. 1g). Second, the system's state become more similar to its past state because of the slowing down, meaning that the time series becomes more auto-correlated (Fig. 1j, Kleinen et al. 2003). 
Transcritical

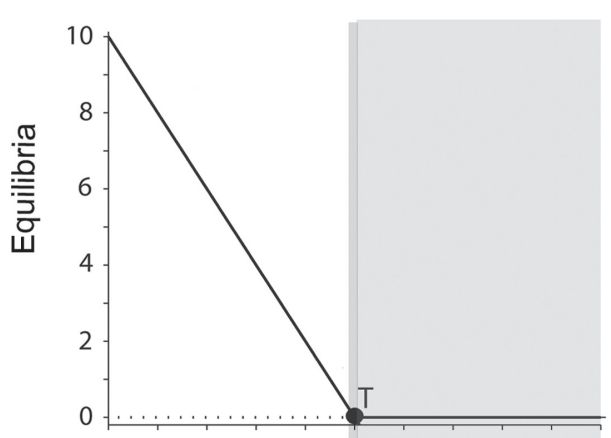

\section{Slowing-down}

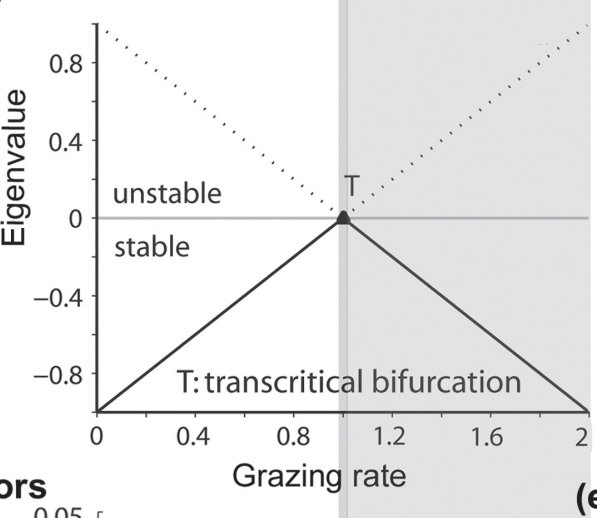

Indicators

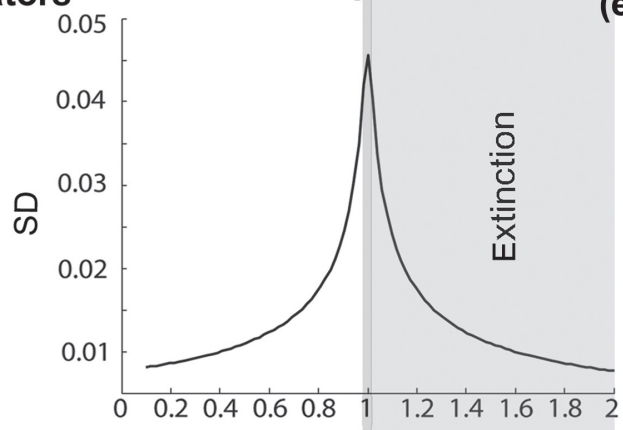

(e)

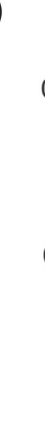

(a)

(c)
Hopf

(b)

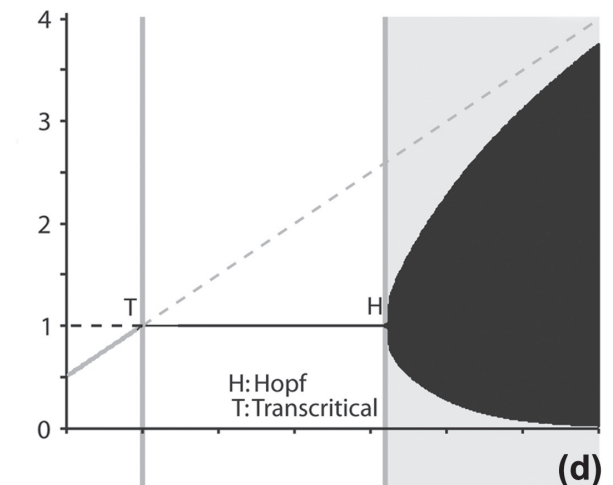

(d)

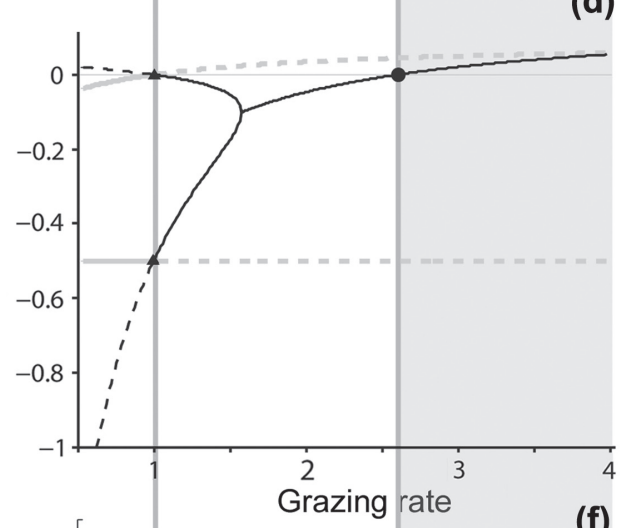

(g)
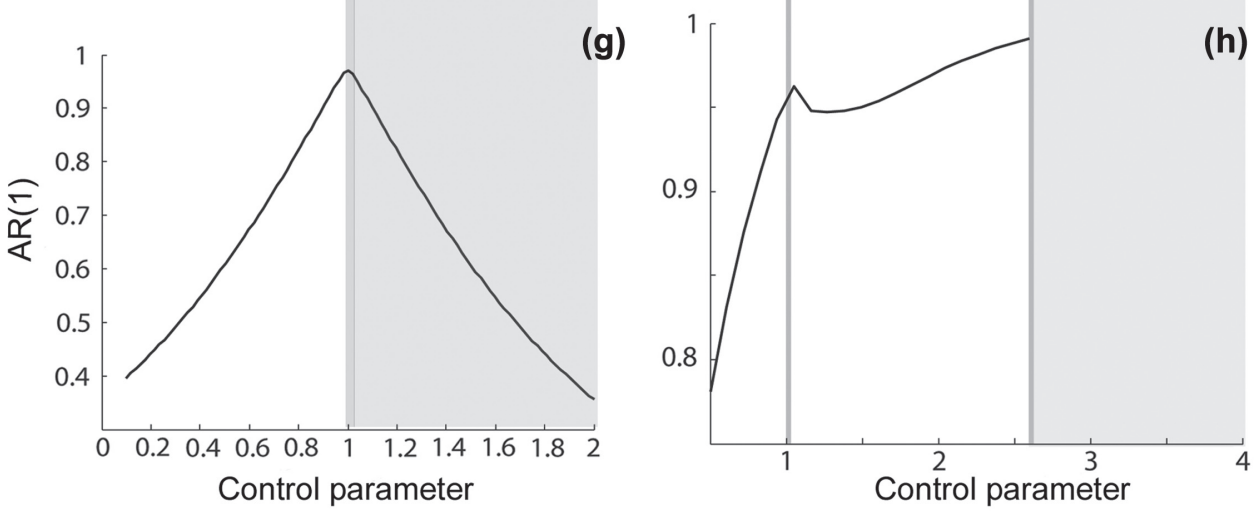

Figure 2. Behavior of the indicators before different types of bifurcation points. Left: transcritical bifurcation; right: hopf bifurcation. First row: model equilibria depending on the control parameter ( $c$ for the transcritical transition and $K$ for the Hopf transition; see Table 1 for parameter definition), second row: dominant eigenvalue of the system, third row: standard deviation, fourth row: autocorrelation at lag one.

2004, Scheffer et al. 2009), two of the questions we would like to address with generic early warning signals are: first, 'what' is going to happen (i.e. what type of ecosystem response is to be expected), and second, 'when' is it going to happen? Our analysis confirms the theoretical expectation that the generic early warnings cannot answer the 'what'question as they behave in a similar way along a range of transitions. This implies that the early warning signals lack 'specific' indicator value. Thus, slowing down and its derived indicators, variance and auto-correlation, may occur, at least 
theoretically, in a broad class of situations even when no catastrophic threshold exists.

The indicators, however, do tell us that 'something' is happening. In that sense the indicators are really generic. If there is no obvious external reason for the changes, the generic early warnings indicate that the system is becoming increasingly slower in response to perturbations, because it is approaching some kind of a transition, catastrophic or not. Interestingly, the sharper the response of the indicators, the higher the sensitivity of the system to changing conditions seems to be (compare panels b, c and e, f of Fig. 1). Whether following changes in the indicators might be useful to compare different instances of the same system to identify the ones that are at a higher risk of undergoing a transition is a topic that deserves further investigation.

The fact that generic early warnings tell us less than we thought has management and policy-related implications. A legitimate question to ask is whether, from a precautionary point of view, it may be worth reacting to any indicator signal to prevent the occurrence of a transition, even if turns out not to be catastrophic. Similar questions have been raised regarding the climate change issue: given the uncertainty of the issue (temperature increase), should we act now (i.e. reduce emission) or should we wait to know more (O'Neill 2008, O'Neill and Sanderson 2008)? Both alternatives may bear a cost, either of the action itself or of the additional climate change in case of no action (O'Neill 2008, O'Neill and Sanderson 2008). Similarly, erroneous projections of ecosystem responses can have significant costs and socio-economic-ecological consequences, either because we take costly actions to prevent a shift when there is none, or because we fail to predict a shift. The question of what policy should be undertaken requires examining the cost-benefit balance (or cost implications) of the two alternative scenarios. A socio-economic analysis of ecosystems that exhibit catastrophic shift has shown that the optimum shared use of the ecosystem tends to be very close to the collapse threshold, a very risky situation since just a small perturbation or imperfect information may lead to a shift (Scheffer et al. 2000). This result stresses how important it is to have some insights about the dynamics of the ecosystem.

In any case, it would still be extremely useful to identify indicators specific to catastrophic shifts, i.e. specific to cases where a system exhibits alternative stable states and hysteresis. Thus, the important question is whether this may be possible at all.

Previous studies have shown that there are ways to gauge whether a catastrophic shift is to be expected in an ecosystem, like past observations of abrupt changes, multimodal distributions of different states, or the presence of ecological mechanisms that may lead to bistability validated by models (Scheffer and Carpenter 2003). However, all these methods require detailed knowledge either on the history of the ecosystem or on its underlying ecological mechanisms.

An alternative way might be to systematically compare the behavior of the existing indicators along different transition types and search for differences in their behaviors. We also need a better knowledge about the cases where the generic early warning signals may fail to announce an approaching shift (Brock and Carpenter 2010, Carpenter and Brock 2010, Hastings and Wysham 2010, Dakos et al. 2011). Combining generic indicators with indicators specific to transition types as well as knowledge on ecosystem properties, even derived from coarse ecological data, could be part of the solution to gauging 'what' transition is about to happen. Inevitably, such direction will lead to new indicators that will match specific types of transitions.

The next challenge is to address the question of 'when' a shift is going to happen. All currently available indicators change gradually and they do not provide a measure of the distance to the transition. For the moment, it seems that most of the indicators will remain relative measures, making sense only in the presence of a baseline. Due to statistical limitations, a trend in a single indicator is likely to be insufficient to determine the proximity to a transition in real ecosystems (Biggs et al. 2009). Therefore combining several indicators to confidently detect an approaching transition (Guttal and Jayaprakash 2009, Drake and Griffen 2010, Dakos et al. 2011), or using both time series and spatial information if available (Rietkerk et al. 2004, Kéfi et al. 2007, 2011) can be a promising way forward. Also combining early warning signals with predictions of mechanistic models may be helpful (Lenton 2011). While uncertainties in the model parameters may be too large for accurate prediction, combining model predictions with early warning signals may improve the reliability.

Despite the fact that generic early warning signals do not provide specific information about the type and the risk of an upcoming transition, testing these ideas and their applicability can still prove beneficial for ecosystem management. Recent studies have provided encouraging results (Dakos et al. 2008, Drake and Griffen 2010, Carpenter et al. 2011, Veraart et al. 2012), and more effort must be devoted to confront these indicators with real data (Andersen et al. 2009). We hope that this work will stimulate further research aiming at devising indicators specific to catastrophic shifts, whose occurrence may be extremely important for the livelihood of people living in the ecosystems threatened.

Acknowledgements - The research of SK and MR has received funding from the European Union's Seventh Framework Programme (FP7/2007-2013) under grant agreement no. 283068 (CASCADE). Part of SK's research was also funded by a Humboldt fellowship from the Alexander von Humboldt foundation. The research of MR is supported by the Netherlands Organization of Scientific Research (NWO), the ERA-Net on Complexity through the project RESINEE (Resilience and interaction of networks in ecology and economics). VD, MS and $\mathrm{EvN}$ are supported by an ERC advanced grant awarded to MS.

\section{References}

Andersen, T. et al. 2009. Ecological thresholds and regime shifts: approaches to identification. - Trends Ecol. Evol. 24: 49-56.

Biggs, R. et al. 2009. Turning back from the brink: detecting an impending regime shift in time to avert it. - Proc. Natl Acad. Sci. USA 106: 826-831. 
Brock, W. A. and Carpenter, S. R. 2010. Interacting regime shifts in ecosystems: implication for early warnings. - Ecol. Monogr. 80: 353-367.

Carpenter, S. and Brock, W. A. 2006. Rising variance: leading indicator of ecological transition. - Ecol. Lett. 9: 311-318.

Carpenter, S. R. and Brock, W. A. 2010. Early warnings of regime shifts in spatial dynamics using the discrete Fourier transform. - Ecosphere 1: art10.

Carpenter, S. R. et al. 2011. Early warnings of regime shifts: a whole ecosystem experiment. - Science 332: 1079-1082.

Chisholm, R. A. and Filotas, E. 2009. Critical slowing down as an indicator of transitions in two-species models. - J. Theor. Biol. 257: 142-149.

Dakos, V. et al. 2008. Slowing down as an early warning signal for abrupt climate change. - Proc. Natl Acad. Sci. USA 105: $14308-14312$.

Dakos, V. et al. 2010. Spatial correlation as leading indicator of catastrophic shifts. - Theor. Ecol. 3: 163-174.

Dakos, V. et al. 2011. Slowing down in spatially patterned ecosystems at the brink of collapse. - Am. Nat. 177: E153-E166.

Dakos, V. et al. 2012. Robustness of variance and autocorrelation as indicators for critical slowing down. - Ecology 93: 264-271.

Diamond, J. 2004. Collapse: how societies choose to fail or survive. - Viking adult, New York.

Drake, J. M. and Griffen, B. D. 2010. Early warning signals of extinction in deteriorating environments. - Nature 467: 456-459.

Folke, C. et al. 2004. Regime shifts, resilience and biodiversity in ecosystem management. - Annu. Rev. Ecol. Syst. 35: $557-581$.

Gilmore, R. 1981. Catastrophe theory for scientists and engineers. - Wiley.

Guttal, V. and Jayaprakash, C. 2009. Spatial variance and spatial skewness: leading indicators of regime shifts in spatial ecological systems. - Theor. Ecol. 2: 3-12.

Hastings, A. and Wysham, D. B. 2010. Regime shifts in ecological systems can occur with no warning. - Ecol. Lett. 13: 464-472.

Holling, C. S. 1973. Resilience and stability of ecological systems. - Annu. Rev. Ecol. Syst. 4: 1-23.

Kéfi, S. et al. 2007. Spatial vegetation patterns and imminent desertification in Mediterranean arid ecosystems. - Nature 449: 213-217.

Kéfi, S. et al. 2011. Robust scaling in ecosystems and the meltdown of patch size distributions before extinction. - Ecol. Lett. 14: 29-35.

Kleinen, T. et al. 2003. The potential role of spectral properties in detecting thresholds in the Eath system: application to the thermohaline circulation. - Ocean Dynamics 53: 53-63.

Kuehn, C. 2011. A mathematical framework for critical transitions: bifurcations, fast-slow systems and stochastic dynamics. - Physica D 240: 1020-1035.

Lenton, T. M. 2011. Early warning of climate tipping points. - Nat. Climate Change 1: 201-209.

Lim, J. and Epueranu, B. I. 2011. Forecasting a class of bifurcations: theory and experiment. - Phys. Rev. E 83: 016203.

Lotka, A. J. 1925. Elements of physical biology (reprinted in 1956). - Dover, New York.

Supplementary material (available online as Appendix O20838 at < www.oikosoffice.lu.se/appendix >). Appendix A1.
May, R. M. 1977. Thresholds and breakpoints in ecosystems with a multiplicity of stable states. - Nature 269: 471-477.

Noy-Meir, I. 1975. Stability of grazing systems an application of predator pey graphs. - J. Ecol. 63: 459-482.

Oborny, B. et al. 2005. Dynamics of populations on the verge of extinction. - Oikos 109: 291-296.

O'Neill, B. C. 2008. Learning and climate change: an introduction and overview. - Climatic Change 89: 1-6.

O'Neill, B. C. and Sanderson, W. 2008. Population, uncertainty and learning in climate change decision analysis. - Climatic Change 89: 87-123.

Peters, D. P. C. and Pielke, R. A. 2004. Cross-scale interactions, nonlinearities, and forecasting catastrophic events. - Proc. Natl Acad. Sci. USA 101: 15130-15135.

Rietkerk, M. et al. 1996. Sahelian rangeland development: a catastrophe? - J. Range Manage. 49: 512-519.

Rietkerk, M. et al. 2004. Self-organized patchiness and catastrophic shifts in ecosystems. - Science 305: 1926-1929.

Rosenzweig, M. L. 1971. Paradox of enrichment - destabilization of exploitation ecosystems in ecological time. - Science 171: 385-388.

Scheffer, M. and Carpenter, S. R. 2003. Catastrophic regime shifts in ecosystems: linking theory to observation. - Trends Ecol. Evol. 18: 648-656.

Scheffer, M. et al. 1997. Seasonal dynamics of Daphnia and algae explained as a periodically forced predator-prey system. - Oikos 80: 519-532.

Scheffer, M. et al. 2000. Socioeconomic mechanisms preventing otimum use of ecosystem services: an interdisciplinary theoretical analysis. - Ecosystems 3: 451-471.

Scheffer, M. et al. 2001. Catastrophic shifts in ecosystems. - Nature 413: 591-596.

Scheffer, M. et al. 2009. Early warning signals for critical transitions. - Nature 461: 53-59.

Sornette, D. 2002. Predictability of catastrophic events: material rupture, earthquakes, turbulence, financial crashes and human birth. - Proc. Natl Acad. Sci. USA V99 SUPP1: 2522-2529.

Sornette, D. 2003. Critical phenomena in natural sciences, chaos, fractals, self-organization and disorder: concepts and tools. - Springer Series in Synergetics.

Stenseth, N. C. et al. 1997. Population regulation in snowshoe hare and Canadian lynx: asymmetric food web configurations between hare and lynx. - Proc. Natl Acad. Sci. USA 94: 5147-5152.

Strogatz, S. H. 1994. Nonlinear dynamics and chaos with applications to physics, biology, chemistry and engineering. - Perseus Books.

Thompson, J. M. T. and Stewart, H. B. 2002. Nonlinear dynamics and chaos, 2nd edn. - Wiley.

Thompson, J. M. T. and Sieber, J. 2011. Predicting climate tipping as a noisy bifurcation: a review. - Int. J. Bifurcation Chaos 21: 399-423.

Van Nes, E. H. and Scheffer, M. 2007. Slow recovery from perturbations as a generic indicator of a nearby catastrophic shift. - Am. Nat. 169: 738-747.

Veraart, A. J. et al. 2012. Recovery rates reflect distance to a tipping point in a living system. - Nature 481: 357-359.

Wissel, C. 1984. A universal law of the characteristic return time near thresholds. - Oecologia 65: 101-107. 